\title{
JOINT SYNCHRONIZATION AND SYMBOL DETECTION IN ASYNCHRONOUS DS-CDMA SYSTEMS
}

\author{
Francesc Rey, Gregori Vázquez, Jaume Riba \\ Department of Signal Theory and Communications, Polytechnic University of Catalonia \\ UPC Campus Nord - Mòdul D5, c/Jordi Girona 1-3, 08034 Barcelona (Spain) \\ e-mail:\{frey,gregori,jriba\}@gps.tsc.upc.es
}

\begin{abstract}
Design accurate estimators which also consider the noise term in low $S N R$ scenarios is paramount to achieve optimal solutions and to obtain precise symbol detectors. Particularly, this paper estimates the propagation delays focusing on asynchronous $D S$ - $C D M A$ systems. The proposed Minimum Conditioned Variance ( $M C V)$ is the choice in noisy environments, implementing the best linear detector of the transmitted symbols under a minimum meansquare error criterion. The result is an estimator that improves the conditional $M L(C M L)$ solution when noise is not negligible, and attains the derived Gaussian Unconditional Cramér-Rao Bound (UCRB) in the whole EbNo range as classical Gaussian Unconditional $M L(U M L)$ does. Consequently, the proposed $M C V$ estimator, becomes an optimal quadratic solution achieving similar features than $U M L$ in a straightforward way, and with no assumptions on the signal statistics.
\end{abstract}

\section{INTRODUCTION}

In digital communications, the knowledge of certain parameters as for example the phase and carrier frequency or the propagation delay, are paramount to get a reliable detection of the transmitted symbols. Focusing on multi-user $D S-C D M A$ systems, an accurate estimation of the propagation delays for all users is essential. Otherwise, the performance of the multi-user detector is rapidly decreased by means of multiple-access interference $(M A I)$, as has been widely studied in the literature [1], [2]. Accordingly, this paper addresses a multi-parametric estimator intended for the multi-user synchronization and symbol detection, with high performance in low $S N R$ scenarios. Nevertheless, the proposed algorithm is not restricted to multi-user synchronizers, and can be also extended to other estimation problems, like frequency synchronization in OFDM and MultiCarrier schemes.

Maximum Likelihood ( $M L)$ formulation has been usually employed to design timing estimators. Classically, Unconditional $M L(U M L)$ algorithms have been developed in

This work has been supported by: TIC98-0412, TIC98-0703, TIC99-0849 (CICYT) and CIRIT/Generalitat de Catalunya 1998SGR-00081. the field of digital communications modeling the transmitted symbols as stochastic processes. Nevertheless, in order to obtain feasible mathematical expressions, $U M L$ estimators make some assumptions on the gaussianity of signal statistics, which is known to be a non-realistic assumption in digital communications, or assumptions on low $S N R$, which leads to self-noise appreciable when the noise term is negligible. Consequently, the restrictions on UML motivated the introduction of deterministic or conditional $M L$ $(C M L)$, which considers the transmitted symbols as deterministic unknown parameters. This formulation has been applied by Stoica and Nehorai [3] in sensor array processing to perform $D O A$ estimation, and more recently the same principle has been applied to frequency and timing estimation [4]-[7]. The $C M L$ solution does not present self-noise, is robust in near-far scenarios, and provides a high performance at high SNR's. Nevertheless it is not an optimal solution in noisy scenarios with low $S N R$.

The proposed Minimum Conditioned Variance (MCV) method, addressed in this paper, mitigates the $C M L$ estimation drawbacks at low $S N R$ scenarios considering the impact of the noise, and becomes the deterministic solution at high $S N R$. Although the derived $M C V$ becomes biased, the bias value can be estimated and next subtracted to obtain an unbiased estimator. The result is an estimator that attains the lower Gaussian Unconditional Cramér-Rao Bound $U C R B$ in the whole EbNo range, as Gaussian $U M L$ does. Accordingly, $M C V$ becomes an optimal quadratic estimator with no assumptions on the signal statistics.

This paper is organized as follows. Next section describes the discrete-time signal model, and obtains a structured matrix expression containing the parameters to estimate. Section 3 describes the $C M L$ formulation and justifies under which conditions the deterministic criterion does not become feasible. Afterwards, section 4 introduces the Minimum Conditioned Variance method as choice, and derives its gradient expression. Furthermore, a detailed study of the proposed estimator shows it is biased and consequently a modified unbiased estimator is proposed. Next, section 5 derives the $U C R B$ which is used as a benchmark, at high and low $S N R$ 's, to the performance of the proposed multiuser delay estimator. Finally last section presents some simulation results proving the proposed $M C V$ outperforms $C M L$, attaining the $U C R B$ and reducing the Bit-Error Rate $B E R$ in symbol detection. 


\section{DISCRETE-TIME SIGNAL MODEL}

The described model considers a $K$ user asynchronous $D S$ $C D M A$ system operating in a multipath environment. The received signal contains the superposition of $K$ active users:

$$
r(t)=\sum_{k=1}^{K} s^{k}\left(t-\tau_{k}\right)+w(t)
$$

where $s^{k}(t)$ denotes the $k$-user received baseband signal, $\tau_{k}$ its the propagation delay, and $w(t)$ represents the received AWGN noise term with zero mean and variance $\sigma_{w}^{2}$. For each user the received baseband signal is modeled as:

$$
s^{k}(t)=\sum_{n=-\infty}^{\infty} d_{n}^{k} e^{j \theta_{k}} g^{k}(t-n T)
$$

where $g^{k}(t)$ represents the $k$-user received signature, $T$ is the bit duration, $d_{n}^{k}$ are the transmitted information bits, and $\theta_{k}$ the received carrier phase. Moreover, considering the presence of a propagation multipath channel with baseband impulse response $h^{k}(t)$, the $k$-user received signature is given by a distorted version of the transmitted spreading waveform $c^{k}(t)$ as:

$$
g^{k}(t)=c^{k}(t) * h^{k}(t)
$$

Finally the received signal as a function of the user's signatures is given by:

$$
r(t)=\sum_{k=1}^{K} \sum_{n=-\infty}^{\infty} d_{n}^{k} e^{j \theta_{k}} g^{k}\left(t-n T-\tau_{k}\right)+w(t)
$$

The algorithm is derived in a discrete-time signal model by sampling the received waveform at $N_{s c}$ samples per chip. Choosing the sampling frequency as $f_{s}=1 / T_{s}$, where $T_{s}$ is the sampling period, and collecting $2 M+1$ samples of $r\left(n T_{s}\right)$, the vector $\mathbf{r}$ can be defined as:

$$
\mathbf{r}=\left[\begin{array}{lllll}
r\left(-M T_{s}\right) & \ldots & r(0) & \ldots & r\left(M T_{s}\right)
\end{array}\right]^{T}
$$

At this point equation (4) can be expressed following the matrix signal model:

$$
\mathbf{r}=\mathbf{A}(\tau) \mathbf{x}+\mathbf{w}^{*)}
$$

The set of unknown parameters (i.e. the transmitted symbols and phase errors) for $k$-user define the vector $\mathbf{x}^{k}$ :

$$
\mathbf{x}^{k}=\left[\begin{array}{lllll}
d_{-L}^{k} e^{j \theta_{k}} & \ldots & d_{0}^{k} e^{j \theta_{k}} & \ldots & d_{L}^{k} e^{j \theta_{k}}
\end{array}\right]^{T}
$$

where the number of transmitted symbols $N_{s}=2 L+1$. Finally, stacking all users, the nuisance parameter vector $\mathbf{x}$ is defined as follows:

$$
\mathbf{x}=\left[\begin{array}{llll}
\mathbf{x}^{1^{T}} & \mathbf{x}^{2^{T}} & \ldots & \mathbf{x}^{K^{T}}
\end{array}\right]^{T}
$$

*)The channel coefficients are assumed to be known or previously estimated (e.g. [8])
On the other hand the model transfer matrix, denoted as $\mathbf{A}(\tau)^{\dagger)}$, contains the user signatures, and the parameters to estimate $\tau_{k}$ :

$$
\begin{aligned}
\mathbf{A}=\mathbf{A}(\tau)= & {\left[\begin{array}{lll}
\mathbf{A}^{1}\left(\tau_{1}\right) & \mathbf{A}^{2}\left(\tau_{2}\right) \ldots \mathbf{A}^{K}\left(\tau_{K}\right)
\end{array}\right] } \\
\mathbf{A}^{k}\left(\tau_{k}\right)= & {\left[\begin{array}{lll}
\mathbf{a}_{0}^{k} & \mathbf{a}_{1}^{k} \ldots \mathbf{a}_{N_{s}-1}^{k}
\end{array}\right] } \\
\mathbf{a}_{n}^{k}= & {\left[g^{k}\left(-M T_{s}-n T-\tau_{k}\right) \ldots\right.} \\
& \left.g^{k}\left(M T_{s}-n T-\tau_{k}\right)\right]^{T}
\end{aligned}
$$

where the columns of $\mathbf{A}^{k}\left(\tau_{k}\right)$ are scrolled versions of the $k$-user signature delayed $\tau_{k}$.

A more detailed model of matrix $\mathbf{A}^{k}\left(\tau_{k}\right)$ will be constituted by the product of two matrices:

$$
\mathbf{A}^{k}\left(\tau_{k}\right)=\mathbf{H}^{k}\left(\mathbf{h}_{\mathbf{k}}\right) \mathbf{C}^{k}\left(\tau_{k}\right)
$$

Matrix $\mathbf{H}^{k}\left(\mathbf{h}_{\mathbf{k}}\right)$ is a Sylvester or convoluting matrix modeling the channel distortion, whose columns are the $k$-user impulsional channel response coefficients. On the other hand, matrix $\mathbf{C}^{k}\left(\tau_{k}\right)$ will be obtained by the $k$-user spreading code delayed $\tau_{k}$

\section{THE CML FORMULATION}

The cost function in $C M L$ estimation for the signal model in (6) is derived from the joint $M L$ cost function that is formulated as:

$$
\Lambda(\mathbf{r} / \tau, \mathbf{x})=\frac{1}{\left(\pi \sigma_{w}^{2}\right)^{M}} e^{-\frac{1}{\sigma_{w}^{2}}\|\mathbf{r}-\mathbf{A x}\|^{2}}
$$

The $M L$ function depends on the parameter estimation vector $\tau$ and also on the vector $\mathbf{x}$. Notice that vector $\mathbf{x}$ contains the set of unknown parameters and thus it is necessary to take some considerations on this vector. The joint $\tau, \mathbf{x}$ estimation could be the solution, but it is discarded because it is computationally complex, and alternative algorithms only focusing on the $\tau$ vector estimation are proposed. Classically, $U M L$ solution computes the expectation of the joint $M L$ function with respect to the nuisance parameters:

$$
\Lambda_{U M L}(\mathbf{r} / \tau)=E_{x}\{\Lambda(\mathbf{r} / \tau, \mathbf{x})\}
$$

In general the expectation $E_{x}$ in (12) is quite difficult to obtain, and in practice only an approximation of the likelihood function in low $S N R$ scenarios is approached. Previous limitations motivate the use of the $C M L$ solution. This method considers the nuisance parameters as deterministic, and thus they can be substituted by its estimation keeping fixed $\tau$ vector. The $M L$ estimation of $\mathbf{x}$, when no restrictions are imposed on it, can be obtained as:

$$
\widehat{\mathbf{x}}_{M L}=\mathbf{A}^{\# \mathbf{r}}
$$

where $\mathbf{A}^{\#}$ is the Moore-Penrose pseudo-inverse. Once the nuisance vector $\mathrm{x}$ is estimated, the compressed $M L$ function to maximize, which only depends on the parameter vector $\tau$, is obtained by replacing (13) in (11). And finally the

\footnotetext{
†)Hereafter the dependence on vector $\tau$ will be suppressed for simplicity
} 
derived log-likelihood function to minimize (omitting irrelevant constants) is given by:

$$
\min _{\tau} L_{C M L}(\mathbf{r} / \tau)=\operatorname{tr}\left\{\mathbf{P}_{A}^{\perp} \hat{\mathbf{R}}\right\}
$$

where $\mathbf{P} \frac{1}{A}=\mathbf{I}-\mathbf{A} \mathbf{A}^{\#}$ is the projection matrix onto the orthogonal subspace defined by $\mathbf{A}$, and $\hat{\mathbf{R}}=\mathbf{r r} \mathbf{r}^{\mathbf{H}}$.

To minimize (14) a gradient algorithm may be used. The gradient in conditional $M L$ was derived by Viberg, Ottersten and Kailath [9] in the context of array processing for $D O A$ estimation. In our delay estimation problem this gradient can be expressed as:

$$
g_{c_{\boldsymbol{i}}}=-2 \operatorname{Re}\left\{\left(\mathbf{r}^{H} \mathbf{P}_{A}^{\perp} \mathbf{D}_{i}\right)\left(\mathbf{A}^{\#} \mathbf{r}\right)\right\}
$$

where $\mathbf{D}_{i}=\frac{\partial}{\partial \tau_{i}} \mathbf{A}$.

A more accurate study of the gradient expression shows that it is computed by the product of two terms. The first term is $\left(\mathbf{r}^{H} \mathbf{P}_{A}^{\perp} \mathbf{D}_{i}\right)$ and justifies the proposed algorithm to be self-noise free. Considering a noiseless environment, and the absence of delay errors, vector $\mathbf{r}$ will be contained in the signal subspace generated by the $A$ matrix columns. Thus, the projection matrix $\mathbf{P}_{A}^{\perp}$, which does not appear in the classical unconditional approach, acts as a zero-forcer placed at the output of the derivative matched filter $\mathbf{D}_{i}$. As a result, the estimator ensures in all cases a self-noise free solution: $\left(\mathbf{r}^{H} \mathbf{P}_{A}^{\perp} \mathbf{D}_{i}\right)=0$.

The second term $\left(\mathbf{A}^{\#} \mathbf{r}\right)$ corresponds to the ML estimation of the unconstrained vector $x$. Notice that this expression is the decorrelating detector solution, so the algorithm not only estimates the propagation delay but also implements this sub-optimum detector. The presence of this term justifies the proposed solution to be a robust near-far estimator. Analyzing the signal model (6) it is observed that the received powers can be introduced in the nuisance parameter vector $\mathbf{x}$. Hence, following (13) it is guaranteed that the algorithm will estimate the received power values, justifying the estimator to be insensitive to different power levels.

Nevertheless, the decorrelating detector evidences some difficulties in noisy scenarios. The pseudoinverse, as the ideal zero-forcing solution $Z F$ in equalization, does not take into account the noise term. Accordingly, when the transfer matrix $\mathbf{A}$ eigenvalue spreading, defined as:

$$
\chi=\frac{\lambda_{A_{\max }}}{\lambda_{A_{\min }}}
$$

is large enough, the noise term will be extremely increased, becoming the $C M L$ method an unacceptable solution in low $S N R$ scenarios, which are common in wideband $D S-C D M A$ systems.

\section{MINIMUM CONDITIONED VARIANCE APPROACH}

A novel approach is proposed in this paper considering the impact of the noise in the likelihood function, achieving in consequence a more robust estimator in low SNR scenarios. The Minimum Conditioned Variance approach (MCV) makes the nuisance parameter estimation as the best linear estimation under a minimum variance criterion given an observation vector $\mathbf{r}$. This estimation is:

$$
\begin{gathered}
\widehat{\mathbf{x}}=E[\mathbf{x} / \mathbf{r}]=\boldsymbol{\Gamma} \mathbf{A}^{H}\left(\mathbf{A} \boldsymbol{\Gamma} \mathbf{A}^{H}+\sigma_{w}^{2} \mathbf{I}\right)^{-1} \mathbf{r}=\mathbf{C r} \\
\mathbf{C}=\boldsymbol{\Gamma} \mathbf{A}^{H}\left(\mathbf{A} \boldsymbol{\Gamma} \mathbf{A}^{H}+\sigma_{w}^{2} \mathbf{I}\right)^{-1} \\
\boldsymbol{\Gamma}=E\left\{\mathbf{x x}^{H}\right\}
\end{gathered}
$$

Previous expression belongs to the best linear and nonlinear estimator under Gaussian conditions, and only the best linear estimator under non-Gaussian conditions. The new cost function is derived by substituting (17) in equation (11) and it is given by:

$$
\min _{\tau} L_{M C V}(\mathbf{r} / \tau)=\|\mathbf{r}-\mathbf{A C r}\|^{2}
$$

At high $S N R$ scenarios $\mathbf{C}\left(\sigma_{w \rightarrow 0}^{2}\right)=\mathbf{A}^{\#}$ is the pseudoinverse of $\mathbf{A}$, becoming the $C M L$ solution. On the other hand, when the contribution of $\mathbf{A} \mathbf{\Gamma} \mathbf{A}^{H}$ is negligible in front of $\sigma_{w}^{2} \mathbf{I}, \mathbf{C}$ approaches a bank of matched filters containing all the user signatures: $\mathbf{C}\left(\sigma_{w \rightarrow \infty}^{2}\right)=\sigma_{w}^{-2} \mathbf{\Gamma} \mathbf{A}^{H}$. This second limit is achieved at low $S N R$ when the noise power is much greater than the received signal power for all users. Notice however that, in high near-far scenarios, the elements in $\Gamma$ associated to the most powerful users will be higher than the noise term. Consequently, in scenarios with low $S N R$ and small near-far, the $M C V$ will improve the classical $C M L$ solution, whereas in high near-far scenarios, $M C V$ will remain close to $C M L$

. To minimize (18) we will follow once again a gradient scheme. The gradient expression in $M C V$ is given by:

$$
g_{m c v_{\mathbf{i}}}=-2 R e\left\{\mathbf{r}^{H}(\mathbf{I}-\mathbf{A C})^{H}\left(\mathbf{D}_{i} \mathbf{C}+\mathbf{A} \frac{\partial}{\partial \tau_{i}} \mathbf{C}\right) \mathbf{r}\right\}
$$

It results interesting to analyze the behaviour at high and low $S N R$ scenarios. At high $S N R \mathbf{C} \rightarrow \mathbf{A}^{\#}$, and making use of $\mathbf{P}_{A}^{\perp} \mathbf{A}=0$, the second term in the previous gradient is asymptotically equal to zero: $\mathbf{r}^{H}(\mathbf{I}-\mathbf{A C})^{H} \mathbf{A} \frac{\partial}{\partial r_{i}} \mathbf{C r}=0$. Thus the gradient becomes:

$$
g_{m c v_{i}}\left(\sigma_{w}^{2} \rightarrow 0\right) \simeq-2 R e\left\{\mathbf{r}^{H}(\mathbf{I}-\mathbf{A C})^{H}\left(\mathbf{D}_{i} \mathbf{C}\right) \mathbf{r}\right\}
$$

Likewise, at low $S N R$ 's $\mathbf{C} \rightarrow \sigma^{-2} \boldsymbol{\Gamma} \mathbf{A}^{H}$, and the two components in the gradient (19) supply the same value. Hence, the asymptotic gradient derived in noisy environments corresponds with:

$$
g_{m c v_{i}}\left(\sigma_{w}^{2} \rightarrow \infty\right) \simeq-\frac{4}{\sigma_{w}^{2}} \operatorname{Re}\left\{\mathbf{r}^{H} \mathbf{D}_{i} \Gamma \mathbf{A}^{H} \mathbf{r}\right\}
$$

Notice that the second term can be dropped in both cases without loosing information by the gradient.

Finally, for the special case when there is only one parameter to estimate, e.g. timing or frequency estimation in linear and non-linear modulations, another argument to eliminate the second term is detailed in [7] and next outlined. Considering that vector $\mathbf{r}$ follows a Gaussian distribution, which is known to be a non-realistic assumption in digital communications, the Gaussian $U M L$ cost function becomes ${ }^{\ddagger)}$ :

$$
\begin{gathered}
L_{U M L^{G}}(\mathbf{r} / \tau)=\mathbf{r}^{H} \mathbf{R}^{-1} \mathbf{r} \\
\mathbf{R}=\left(\mathbf{A} \Gamma \mathbf{A}^{H}+\sigma_{w}^{2} \mathbf{I}\right)
\end{gathered}
$$

‡) only applicable if $\mathbf{A}^{H} \mathbf{A}$ does not depend on the parameter to estimate 
and the Gaussian $U M L$ gradient in previous equation is given by:

$$
g_{u m l_{i}}=-2 \operatorname{Re}\left\{\mathbf{r}^{H}(\mathbf{I}-\mathbf{A C})^{H} \mathbf{D}_{i} \mathbf{C r}\right\}
$$

Comparing last equation with (19), a further justification for removing the second term is obtained. Accordingly, in uni-parametric estimators, and assuming a Gaussian distribution for the transmitted symbols, the $M C V$ gradient becomes the Gaussian $U M L$ gradient. Nevertheless, in multiparametric estimators, the Gaussian $U M L$ cost function becomes more complex:

$$
L_{U M L} G(\mathbf{r} / \tau)=\ln |\mathbf{R}|+\mathbf{r}^{H} \mathbf{R}^{-1} \mathbf{r}
$$

Notice a new term $\ln |\mathbf{R}|$, which becomes constant in the uniparametric estimators when $\mathbf{A}^{H} \mathbf{A}$ does not depend on the parameter to estimate, is introduced. The gradient expression, derived in [3] cannot be identified with (19) anymore.

After the previous analysis, the $M C V$ gradient can be asymptotically rewritten as:

$$
g_{m c v_{i}} \approx-2 \operatorname{Re}\left\{\mathbf{r}^{H}(\mathbf{I}-\mathbf{A C})^{H} \mathbf{D}_{i} \mathbf{C r}\right\}
$$

A more accurate analysis of the previous gradient shows it is biased. It can be seen that in the absence of timing errors the gradient does not become the null vector. Therefore, the bias expression can be obtained computing the gradient expected value when the estimated timing vector equals the real timing vector:

$$
\begin{aligned}
\text { Bias }_{i} & =\left.E\left\{g_{m c v_{i}}\right\}\right|_{\hat{\tau}=\tau} \\
& =-2 \operatorname{Re}\left\{\operatorname{Tr}\left\{\boldsymbol{\Gamma} \mathbf{A}_{\tau}^{H}\left(\mathbf{I}-\mathbf{A}_{\tau} \mathbf{C}_{\tau}\right)^{H} \mathbf{D}_{i_{\tau}}\right\}\right\}
\end{aligned}
$$

denoting $\mathbf{A}_{\tau}, \mathbf{C}_{\tau}, \mathbf{D}_{i_{\tau}}$, the dependence of matrices on $\tau$. Unfortunately previous expression cannot be computed by the estimator because the real timing vector $\tau$ is not a priori known. Nevertheless, the gradient expected value close to the real timing vector does not depend on the absolute timing error $\tau-\hat{\tau}$. Hence, an accurate bias estimation can be obtained if the estimated timing vector is used to compute (26):

$$
\widehat{B i a s}_{i}=-2 \operatorname{Re}\left\{\operatorname{Tr}\left\{\boldsymbol{\Gamma} \mathbf{A}_{\hat{\tau}}^{H}\left(\mathbf{I}-\mathbf{A}_{\hat{\tau}} \mathbf{C}_{\hat{\tau}}\right)^{H} \mathbf{D}_{i_{f}}\right\}\right\}
$$

As a result, an unbiased estimation of $\tau$ vector can be obtained according to a modified gradient where the bias is subtracted:

$$
g_{m c v_{i}}^{\text {uniased }}=-2 \operatorname{Re}\left\{\mathbf{r}^{H}(\mathbf{I}-\mathbf{A C})^{H} \mathbf{D}_{i} \mathbf{C r}\right\}-\widehat{\text { ias }_{i}}
$$

\section{PERFORMANCE ANALYSIS}

This section derives the Gaussian Unconditional CramérRao Bound (UCRB) to compare it with the proposed $C M L$ and $M C V$ multi-user delay estimators analyzing its performance. As it is shown in [3] the UCRB is a valid lower bound for the variance of any consistent estimator based on the data sample covariance matrix.

As derived in [10] the $i j$ th Fisher Information Matrix (FIM) element can be obtained as:

$$
\left\{F I M_{u}\right\}_{i j}=\operatorname{Tr}\left\{\mathbf{R}^{-1} \mathbf{R}_{i} \mathbf{R}^{-1} \mathbf{R}_{j}\right\}
$$

where:

$$
\begin{gathered}
\mathbf{R}=E\left\{\mathbf{r} \mathbf{r}^{H}\right\}=\mathbf{A} \mathbf{\Gamma} \mathbf{A}^{H}+\sigma_{w}^{2} \mathbf{I} \\
\mathbf{R}_{i}=\frac{\partial}{\partial \tau i} \mathbf{R}
\end{gathered}
$$

Focusing on our estimation problem, assuming that the noise power is a priori known. (which is considered in the $M C V$ case), and modeling the transmitted symbols to be zero mean independent random variables (i.e. $\Gamma$ is a diagonal matrix) $\mathbf{R}_{i}$ results:

$$
\begin{gathered}
\mathbf{R}_{i}=\sigma_{x i}^{2}\left(\mathbf{D}_{i} \mathbf{A}^{H}+\mathbf{A D}_{i}^{H}\right) i=1 \ldots N_{s} \\
\mathbf{D}_{i}=\frac{\partial}{\partial \tau i} \mathbf{A}
\end{gathered}
$$

which can be substituted into (29) to obtain the UCRB.

\section{SIMULATION RESULTS}

To evaluate the $C M L(15)$ and $M C V(28)$ estimators its performance was compared computing the Root-Mean Square Error $(R M S E)$ in the timing delay estimation, and the Biterror Rate $(B E R)$ in the symbol detection. Simulations were done considering 5 users, the spreading codes were Gold sequences with 7 chips per bit, the pulse shaping was a square-root raised cosine pulse with roll-off factor equal to 0.5 and the considered modulation was BPSK, and the oversampling factor was $N_{s c}=2$. Denoting $B_{L}$ as the equivalent noise loop bandwidth, this parameter is related with the number of transmitted symbols as [12]:

$$
B_{L} T=\frac{1}{2 N_{s}}
$$

and the UCRB lower bound is usually written as a function this bandwidth factor.

Figure 1 compares the proposed $M C V$ versus the classical $C M L$ algorithm, and compares the $R M S E$ with the derived $U C R B$ lower bound assuming that the noise power $\sigma_{w}^{2}$ is a priori known (29) - (31). A low $S N R$ scenario with near-far $N F=0$ and only one path per user on $A W G N$ (i.e. no channel assumption) was simulated. As it can be seen in figure 1, due to the high eigenvalue spread, at low $S N R$ the $C M L$ is not an optimal solution and does not achieve the derived $U C R B$. Under those conditions, the proposed $M C V$ outperforms the $C M L$ algorithm and attains the UCRB, becoming a quadratic optimal solution. Figure 1 also illustrates how at high $S N R$ the $M C V$ becomes the $C M L$ solution, and asymptotically both attain the Cramér-Rao Bound.

A second simulation shows the performance of both algorithms in symbol detection, and illustrates once again the importance of $M C V$ in noisy environments. Figure 2 compares the BER according to the ML estimation of vector x (13) considered in $C M L$ estimation, and the MMSE estimator (17) introduced in the $M C V$ approach. In order to illustrate the eigenvalues spread importance, two simulations, using 7 chips per bit spreading codes (associated eigenvalue spreading $\chi: 35$ ) and 15 chips per bit spreading codes (associated eigenvalue spreading $\chi: 6.25$ ), were done. As it can be seen, the higher the eigenvalue spreading is, the worse the $C M L$ solution performs. When the system 


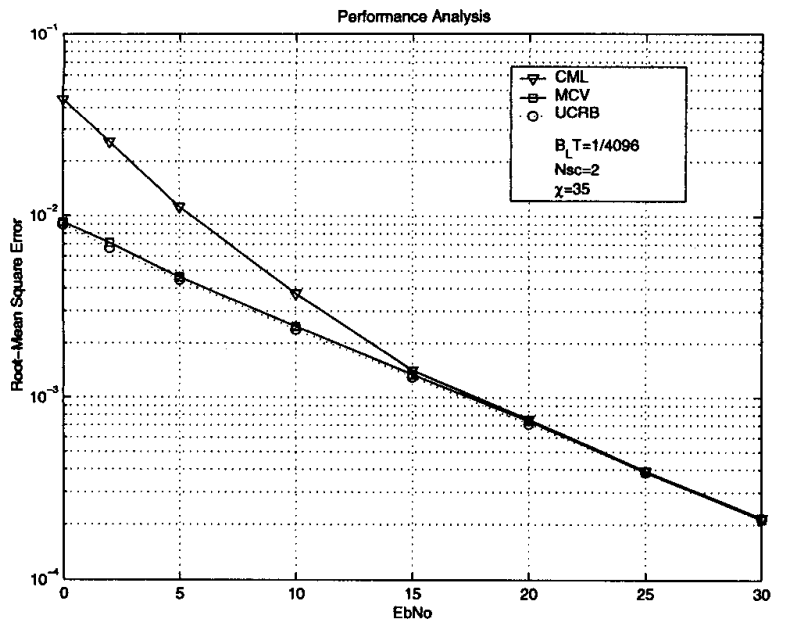

Figure 1: Timing Delay Estimation Error

is working at the limit of its capacity, (i.e. 5 users and spreading factor 7) the noise power is extremely increased by the decorrelating detector, and $C M L$ is not an acceptable solution, while the novel $M C V$ always achieves a better performance.

\section{CONCLUSIONS}

In this paper the $M C V$ algorithm has been introduced in the multiuser propagation delay estimation context. This novel method modifies the classical $C M L$ solution considering the impact of the noise in the Likelihood function compression. Hence, a more robust algorithm in noisy environments when the transference matrix eigenvalue dispersion is large, can be derived.

Simulations have shown $M C V$ outperforms the classical deterministic algorithm in noisy conditions, and it corresponds asymptotically with the $C M L$ at high $S N R$ 's. The mean squared timing error and the bit-error rate at the symbol detection have been used to evaluate this performance. Accordingly, the suggested quadratic estimation technique is shown to be optimal since it attains the UCRB lower bound in the whole EbNo range, becoming a great substitute not only to $C M L$, but also to $U M L$ because it achieves similar features in a straightforward way.

\section{REFERENCES}

[1] E.G. Ström, S. Parkvall, S.L. Miller, B.E. Ottersten, "Propagation delay estimation in asynchronous directsequence code-division multiple access systems", IEEE Trans. on Commun., vol 44, Jan. 1996.

[2] Z.Liu, J. Li, S.L. Miller, "An Efficient Code-Timing Estimator for Receiver Diversity DS-CDMA Systems", IEEE Trans. on Communications, vol 46, Jun. 1998.

[3] P.Stoica, A.Nehorai. "Performance Study of Conditional and Unconditional Direction Of-Arrival Estima-

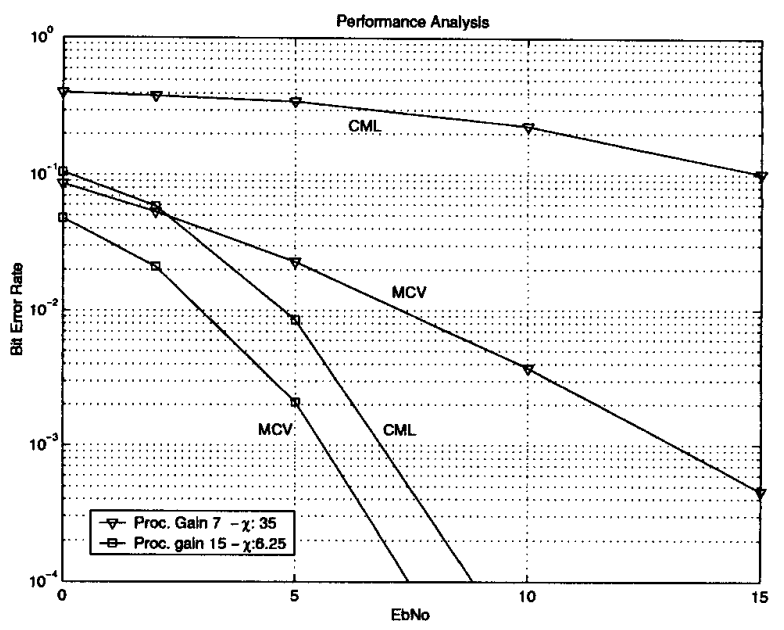

Figure 2: BER Symbol Detection

tion", IEEE Trans. on Acoustics, Speech and Signal Processing, vol.38, October 1990.

[4] J.Riba, G. Vázquez, S. Calvo. "Conditional Maximum Likelihood Frequency Estimation for Staggered Modulations". Proc. of ICASSP'98, Seattle (USA).

[5] J.Riba, G.Vázquez. "CML Timing Recovery", Proc. of ICASSP'99, Phoenix (USA).

[6] J. Riba, J. Sala, G. Vázquez. "Conditional Maximum Likelihood Timing Recovery: Estimators and Bounds". Submitted to IEEE. Trans on Communications 1999.

[7] G. Vázquez, J.Riba. Non-Data-Aided Digital Syncronization. In G. B. Giannakis, Y. Hua, P. Stoica, and L. Tong, editors. Signal Processing Advances in Wireless Communications, volume. II: Trends in Single and Multi-User Systems, chapter. 9. Prentice-Hall, 2000. To be published.

[8] S.E. Bensley, B. Aazhang, "Subspace Based Channel Estimation for Code Division Multiple Access Communication Systems", IEEE Trans. on Communications, vol 44, August 1996.

[9] M.Viberg, B. Ottersten, T.Kailath. "Detection and Estimation in Sensor Arrays Using Weighted Subspace Fitting". IEEE Transactions on Signal Processing, vol 39, November 1991.

[10] B. Ottersten, M. Viberg, P. Stoica, Radar Array Processing. Springer-Verlag, 1993. Chapter: Exact and Large Sample ML Techniques for Parameter Estimation and Detection.

[11] F. Rey, G. Vázquez, J. Riba. "Near-Far Resistant CML Propagation Delay Estimation and Multi-user Detection for Asynchronous DS-CDMA Systems". Proc. of VTC-fall'99, Amsterdam (The Netherlands).

[12] Umberto Mengali, Aldo N. D'Andrea, Synchronization Techniques for Digital Receivers, Plenum Press,1997. 